\title{
Interacting dark sector with transversal interaction
}

\author{
Luis P. Chimento ${ }^{1}$ and Martín G. Richarte ${ }^{1}$ \\ ${ }^{1}$ Departamento de Física, Facultad de Ciencias Exactas y Naturales, \\ Universidad de Buenos Aires and IFIBA, CONICET, \\ Ciudad Universitaria, Pabellón I, Buenos Aires 1428, Argentina
}

\begin{abstract}
We investigate the interacting dark sector composed of dark matter, dark energy, and dark radiation for a spatially flat Friedmann-Robertson-Walker (FRW) background by introducing a threedimensional internal space spanned by the interaction vector $\mathbf{Q}$ and solve the source equation for a linear transversal interaction. Then, we explore a realistic model with dark matter coupled to a scalar field plus a decoupled radiation term, analyze the amount of dark energy in the radiation era and find that our model is consistent with the recent measurements of cosmic microwave background anisotropy coming from Planck along with the future constraints achievable by CMBPol experiment.
\end{abstract}

PACS numbers:

Keywords: linear transversal interaction, dark matter, dark energy, dark radiation, scalar field, early dark energy

\section{INTRODUCTION}

One could consider an exchange of energy between the components of the dark sector, so dark matter not only can feel the presence of dark energy through a gravitational expansion of the universe but also can interact between them [1], [2], [3], [4]. A coupling between dark energy and dark matter changes the background evolution of the dark sector allowing us to constrain any particular kind of interaction and giving rise to a richer cosmological dynamics compared with non-interacting models [1], [5]. One way to extend the insight about the dark matterdark energy interacting mechanism is to explore a bigger picture in which a third component is added, perhaps a weakly interacting radiation term as it occurs within warm inflation paradigm. A step forward for constraining dark matter and dark energy is to use the physic behind recombination or big-bang nucleosynthesis epochs by adding a decoupled radiation term to the dark sector for taking into account the stringent bounds related to the behavior of dark energy at early times [3], 6], [7]. The authors have explored the behavior of a universe filled with three interacting components, i.e., dark energy, dark matter, and dark radiation [8] plus a decoupled radiation term (Model I). Then, they have extended the aforesaid scheme by considering a more realistic model with dark matter coupled to a scalar field plus a decoupled radiation (Model II) [9]. We perform a cosmological constraint using the updated Hubble data 10], bounds for early dark energy [11], 12], [13], [14] and compare our constraints on cosmological parameters with the bounds reported by Planck measurements [15] and WMAP-9 project [16] .

\section{MODEL I: ENLARGED DARK SECTOR}

We consider a spatially flat FRW spacetime filled with three interacting fluids, namely, dark energy, dark matter and radiation so that the dynamics of the model is given by the Friedmann and conservation equations,

$$
\begin{gathered}
3 H^{2}=\rho=\rho_{x}+\rho_{m}+\rho_{r} \\
\dot{\rho}+3 H\left(\rho_{x}+p_{x}+\rho_{m}+p_{m}+\rho_{r}+p_{r}\right)=0
\end{gathered}
$$

where $H=\dot{a} / a$ is the Hubble expansion rate and $a$ is the scale factor. Introducing the variable $\eta=\ln \left(a / a_{0}\right)^{3}$, with $a_{0}$ the present value of the scale factor and ${ }^{\prime} \equiv d / d \eta$ and assuming linear barotropic equations of state for the fluids, $p_{i}=\left(\gamma_{i}-1\right) \rho_{i}$, with constant barotropic indexes $\gamma_{i}$ being $i=x, m, r$, Eq. (2) can be recast

$$
\rho^{\prime}=-\gamma_{x} \rho_{x}-\gamma_{m} \rho_{m}-\gamma_{r} \rho_{r},
$$

where the $\gamma_{i}$ satisfy the conditions $0<\gamma_{x}<\gamma_{m}<\gamma_{r}$. The interaction terms $3 H Q_{x}\left(\rho, \rho^{\prime}, \eta\right), 3 H Q_{m}\left(\rho, \rho^{\prime}, \eta\right)$ and $3 H Q_{r}\left(\rho, \rho^{\prime}, \eta\right)$ between the components are introduced by splitting (3) into three equations:

$$
\begin{array}{r}
\rho_{x}^{\prime}+\gamma_{x} \rho_{x}=Q_{x}, \\
\rho_{m}^{\prime}+\gamma_{m} \rho_{m}=Q_{m}, \\
\rho_{r}^{\prime}+\gamma_{r} \rho_{r}=Q_{r},
\end{array}
$$

where $Q_{x}+Q_{m}+Q_{r}=0$ to recover the whole conservation equation (3).

We introduce a 3-dimensional internal space where the three interaction terms and barotropic indexes are viewed as vectors. The interaction vector $\mathbf{Q}=\left(Q_{x}, Q_{m}, Q_{r}\right)$ belongs to the interaction plane $\Pi$ generated by $Q_{x}+$ $Q_{m}+Q_{r}=0$ while the barotropic vector $\gamma=\left(\gamma_{x}, \gamma_{m}, \gamma_{r}\right)$ intersects it. At this intersection, we set the coordinate system origin of the internal space see Fig. (11).

After differentiating Eq. (3) and using Eqs. (4)-(6) we have the additional equation

$$
\rho^{\prime \prime}+\gamma \cdot \mathbf{Q}=\gamma_{x}^{2} \rho_{x}+\gamma_{m}^{2} \rho_{m}+\gamma_{r}^{2} \rho_{r} .
$$

Solving the algebraic system of equations in the $\left(\rho_{x}, \rho_{m}, \rho_{r}\right)$ variables (11), (3), and (7), we obtain

$$
\rho_{x}=\frac{\gamma_{m}-\gamma_{r}}{\Delta}\left[\gamma_{m} \gamma_{r} \rho+\left(\gamma_{m}+\gamma_{r}\right) \rho^{\prime}+\rho^{\prime \prime}+\gamma \cdot \mathbf{Q}\right],
$$




$$
\begin{aligned}
\rho_{m} & =\frac{\gamma_{r}-\gamma_{x}}{\Delta}\left[\gamma_{x} \gamma_{r} \rho+\left(\gamma_{x}+\gamma_{r}\right) \rho^{\prime}+\rho^{\prime \prime}+\gamma \cdot \mathbf{Q}\right] \\
\rho_{r} & =\frac{\gamma_{x}-\gamma_{m}}{\Delta}\left[\gamma_{x} \gamma_{m} \rho+\left(\gamma_{x}+\gamma_{m}\right) \rho^{\prime}+\rho^{\prime \prime}+\gamma \cdot \mathbf{Q}\right]
\end{aligned}
$$

where $\Delta=\left(\gamma_{x}-\gamma_{m}\right) \cdot\left(\gamma_{x}-\gamma_{r}\right) \cdot\left(\gamma_{m}-\gamma_{r}\right)$ is the determinant of that system of equations. Equations (8)-(10) represent the straightforward extension of the interacting two-fluid scenario studied in [1]. Following Ref. [1], we replace Eqs. (8)-(10) into (40)-(6) and obtain the third order differential equation, that we call "source equation", for the total energy density;

$$
\begin{gathered}
\rho^{\prime \prime \prime}+\left(\gamma_{x}+\gamma_{m}+\gamma_{r}\right) \rho^{\prime \prime}+\left(\gamma_{x} \gamma_{r}+\gamma_{x} \gamma_{m}+\gamma_{m} \gamma_{r}\right) \rho^{\prime}+ \\
\gamma_{x} \gamma_{m} \gamma_{r} \rho=\gamma_{x} Q_{m} \gamma_{r}+\gamma_{r} Q_{x} \gamma_{m}+\gamma_{m} Q_{r} \gamma_{x}-\gamma \cdot \mathbf{Q}^{\prime} .
\end{gathered}
$$

To simplify the mathematical treatment of the model, we exploit the internal space structure. To this end, we introduce a pair of vectors $\left\{\mathbf{e}_{\mathbf{t}}, \mathbf{e}_{\mathbf{o}}\right\}$ in $\Pi, \mathbf{e}_{\mathbf{t}}$ is orthogonal to the vector $\gamma$, so $\gamma \cdot \mathbf{e}_{\mathbf{t}}=0$, the orthogonal projection of the vector $\gamma$ on $\Pi$ defines the direction of the vector $\mathbf{e}_{\mathbf{o}}$, and a normal vector $\mathbf{n}=(1,1,1)$ to $\Pi$.

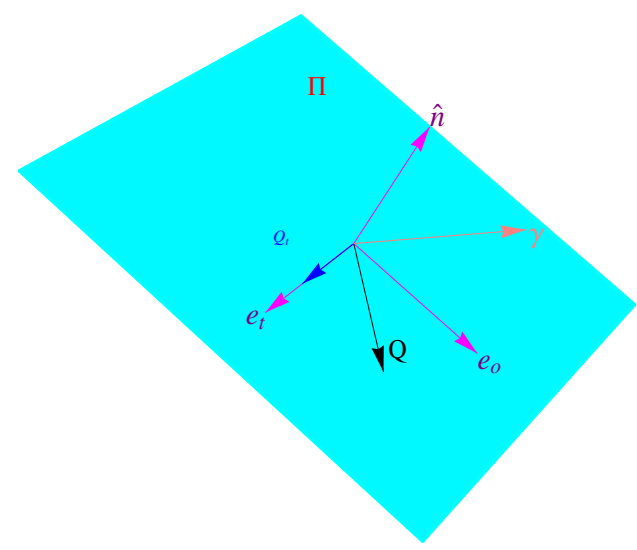

FIG. 1: The plot shows the vector basis $\left\{\mathbf{e}_{\mathbf{t}}, \mathbf{e}_{\mathbf{o}}, \mathbf{n}\right\}$, the interaction plane $Q_{x}+Q_{m}+Q_{r}=0$, the interaction vector $\mathbf{Q}=Q_{t} \mathbf{e}_{\mathbf{t}}+Q_{o} \mathbf{e}_{\mathbf{o}}$ and the vector $\gamma=\gamma_{n} \mathbf{n}+\gamma_{o} \mathbf{e}_{\mathbf{o}}$.

Therefore, $\mathbf{Q}=Q_{t} \mathbf{e}_{\mathbf{t}}+Q_{o} \mathbf{e}_{\mathbf{o}}$, where $Q_{t}$ and $Q_{o}$ are the components of the interaction vector $\mathbf{Q}$ on $\Pi$ and $\mathbf{n} \cdot \mathbf{Q}=$ 0 . Due that the direction of $\mathbf{e}_{\mathbf{t}}$ is the unique with the property of being orthogonal to $\gamma$, we adopt this property as a simple criteria for selecting only those interactions which are collinear with the aforesaid preferred direction in $\Pi$ that we call "transversal interaction", so

$$
\mathbf{Q}_{\mathbf{t}}=Q_{t}\left(\gamma_{m}-\gamma_{r}, \gamma_{r}-\gamma_{x}, \gamma_{x}-\gamma_{m}\right),
$$

ensuring that $\gamma \cdot \mathbf{Q}=0$. The transversal character of the interaction vector (23) removes the terms $\gamma \cdot \mathbf{Q}$ and $\gamma \cdot \mathbf{Q}^{\prime}$ from Eqs. (8)-(11). Besides, the r.h.s. of the Eq. (11) becomes $Q_{t} \Delta$ and the source equation reduces to

$$
\begin{gathered}
\rho^{\prime \prime \prime}+\left(\gamma_{x}+\gamma_{m}+\gamma_{r}\right) \rho^{\prime \prime}+\left(\gamma_{x} \gamma_{r}+\gamma_{x} \gamma_{m}+\gamma_{m} \gamma_{r}\right) \rho^{\prime}+ \\
\gamma_{x} \gamma_{m} \gamma_{r} \rho=Q_{t} \Delta .
\end{gathered}
$$

Once the transversal interaction $\mathbf{Q}_{\mathbf{t}}$ is specified we obtain the energy density $\rho$ by solving the source equation (13) and the component energy densities $\rho_{x}, \rho_{m}$, and $\rho_{r}$ after inserting $\rho$ into Eqs. (8)-(10). We center in a model where the energy exchange is given by transversal interactions which are linearly dependent on $\rho_{x}, \rho_{m}, \rho_{r}$, along with their derivatives up to first order, and $\rho, \rho^{\prime}, \rho^{\prime \prime}, \rho^{\prime \prime \prime}$. Hence, after using Eqs. (8)-(10) one finds that

$$
Q_{t}=\beta_{1} \rho+\beta_{2} \rho^{\prime}+\beta_{3} \rho^{\prime \prime}+\beta_{4} \rho^{\prime \prime \prime}
$$

becomes a linear functional of the basis elements $\rho, \rho^{\prime}$, $\rho^{\prime \prime}, \rho^{\prime \prime \prime}$ (see [1], 8]), and $\beta_{1}, \beta_{2}, \beta_{3}, \beta_{4}$ are four constant parameters. For the interation (14) the source equation (13) turns into a linear differential equation whose general exact solution reads,

$$
\rho=3 H_{0}^{2}\left[\mathcal{A}(z+1)^{3 \gamma_{s}}+\mathcal{B}(z+1)^{3 \gamma_{+}}+\mathcal{C}(z+1)^{3 \gamma_{-}}\right],
$$

where $a^{-1}=z+1$ and $z$ is the cosmological redshift while $\left(\gamma_{s}, \gamma_{-}, \gamma_{+}\right)$are the characteristic roots of the source equation (13), and $\gamma_{s}$ is the minimum of $\left\{\gamma_{s}, \gamma_{-}, \gamma_{+}\right\}$. The $\rho_{i}$ are obtained by inserting Eq. (15) into Eqs. (8)(10) whereas the constants $\mathcal{A}, \mathcal{B}$ and $\mathcal{C}$ are written in term of the density parameters $\Omega_{0 i}=\rho_{0 i} / 3 H_{0}^{2}$ which fulfill the condition $\Omega_{x 0}+\Omega_{r 0}+\Omega_{m 0}=1$ for a flat FRW Universe. Additionally, we will choose $\left(\gamma_{x}, \gamma_{m}, \gamma_{r}\right)=(0,1,4 / 3)$ to recover the three self-conserved cosmic components in the limit of vanishing interaction.

\section{MODEL II: MATTER COUPLED WITH A SCALAR FIELD AND DECOUPLED RADIATION}

Now, we consider a model with matter $\rho_{\mathrm{m}}$ coupled to a scalar field $\rho_{\phi}$ plus a decoupled component $\rho_{\mathrm{r}}$, so that the evolution of the FRW universe is governed by

$$
\begin{gathered}
3 H^{2}=\rho_{\mathrm{t}}=\rho_{\mathrm{m}}+\frac{1}{2} \dot{\phi}^{2}+V(\phi)+\rho_{\mathrm{r}}, \\
\dot{\rho}+3 H\left(\rho_{\mathrm{m}}+\dot{\phi}^{2}\right)=0, \quad \dot{\rho}_{\mathrm{r}}+3 H \gamma_{\mathrm{r}} \rho_{\mathrm{r}}=0, \\
\rho=\rho_{\mathrm{m}}+\frac{1}{2} \dot{\phi}^{2}+V(\phi),
\end{gathered}
$$

where $\rho$ includes all dark components, so $\rho_{\mathrm{t}}=\rho+\rho_{\mathrm{r}}$. We identify the scalar field with a fluid having energy density $\rho_{\phi}=\frac{1}{2} \dot{\phi}^{2}+V(\phi)$, pressure $p_{\phi}=\frac{1}{2} \dot{\phi}^{2}-V(\phi)$ and describe them as a mix of two fluids, namely, $\rho_{\mathrm{sm}}=\dot{\phi}^{2} / 2$ and $\rho_{\mathrm{v}}=V(\phi)$, with equations of state $p_{\mathrm{sm}}=\rho_{\mathrm{sm}}$ and 
$p_{\mathrm{v}}=-\rho_{\mathrm{v}}$ respectively, so $\gamma_{\mathrm{v}}=0, \gamma_{\mathrm{m}}=1, \gamma_{\mathrm{sm}}=2$ and $\gamma_{\mathrm{r}}$ will be estimated later on. Then, $\rho_{\mathrm{sm}}$ can be associated with stiff matter, $\rho_{\mathrm{v}}$ plays the role of vacuum energy, while $\rho_{\mathrm{m}}$ represents a pressureless matter component .

When additionally the matter interacts with the scalar field, we split the Eq. (17) into three balance equations

$$
\begin{gathered}
\rho_{\mathrm{v}}^{\prime}=Q_{\mathrm{v}}, \quad \rho_{\mathrm{m}}^{\prime}+\rho_{\mathrm{m}}=Q_{\mathrm{m}}, \\
\rho_{\mathrm{sm}}^{\prime}+2 \rho_{\mathrm{sm}}=Q_{\mathrm{sm}},
\end{gathered}
$$

so that the whole conservation equation reads

$$
\rho^{\prime}=-\rho_{\mathrm{m}}-2 \rho_{\mathrm{sm}},
$$

while the dynamics of the scalar field is given by the following modified Klein-Gordon (MKG) equation,

$$
\ddot{\phi}+3 H \dot{\phi}+V^{\prime}(\phi)=\frac{-3 H Q_{\mathrm{m}}}{\dot{\phi}} .
$$

Our goal is to build a realistic cosmological model with an early radiation-dominated era. Next, the Universe entered into dark matter era, the radiation becomes subdominant and then follows a dark energy dominated stage at late times. To this end and taking into account that $\gamma=(0,1,2)$, we adopt the transversal interaction

$$
\mathbf{Q}_{\mathbf{t}}=(1,-2,1) Q_{\mathrm{v}} .
$$

By combining Eqs. (19) and (23), we get $Q_{\mathrm{m}}=-2 Q_{\mathrm{v}}=$ $-2 \rho_{\mathrm{v}}^{\prime}=-2(d V / d \phi)(d \phi / d \eta)=-2(d V / d \phi)(d \phi / 3 H d t)$. Thus, the MKG equation (22) becomes $\ddot{\phi}+3 H \dot{\phi}-V^{\prime}(\phi)=$ 0 , showing that $\mathbf{Q}_{\mathbf{t}}$ changes the sign of $V^{\prime}(\phi)$ in the $\mathrm{KG}$ equation. We actually get the transversal interaction leads to $Q_{\mathrm{v}}=V^{\prime}(a)$, so it changes the behavior of the scalar field.

We have built an interacting three fluid model with energy trasnfer (23) defined by Eqs. (18), (21), and $\rho^{\prime \prime}=$ $\rho_{\mathrm{m}}+4 \rho_{\mathrm{sm}}$ obtained after differentiation Eq. (21) and using Eqs. (19)-(20) and (23). By solving this system of equations in the $\left(\rho_{\mathrm{v}}, \rho_{\mathrm{m}}, \rho_{\mathrm{sm}}\right)$-variables, we find

$$
\begin{gathered}
\rho_{\mathrm{v}}=\frac{1}{2}\left[2 \rho+3 \rho^{\prime}+\rho^{\prime \prime}\right] \\
\rho_{\mathrm{m}}=-\left[2 \rho^{\prime}+\rho^{\prime \prime}\right], \quad \rho_{\mathrm{sm}}=\frac{1}{2}\left[\rho^{\prime}+\rho^{\prime \prime}\right] .
\end{gathered}
$$

Following Refs. [1]-[8], we replace Eqs. (24)-(25) into Eq. (19) or (20) and get the source equation for $\rho$ :

$$
\rho^{\prime \prime \prime}+3 \rho^{\prime \prime}+2 \rho^{\prime}=2 Q_{\mathrm{v}} .
$$

Using the general solution of the Eq. (26), that has the form of (15), and inserting it into Eqs. (24)-(25), we have $\rho_{\mathrm{v}}, \rho_{\mathrm{m}}$ and $\rho_{\mathrm{sm}}$. There, we have made the substitution $\rho_{\mathrm{v}} \rightarrow \rho_{\mathrm{x}}, \rho_{\mathrm{m}} \rightarrow \rho_{\mathrm{c}}$, and $\rho_{\mathrm{sm}} \rightarrow \rho_{\mathrm{dr}}$ in order to show explicitly the interaction between these components. Then, $\rho_{\mathrm{x}}, \rho_{\mathrm{c}}$, and $\rho_{\mathrm{dr}}$ will represent the dark energy, dark matter and dark radiation energy densities and, we will assume that $\gamma_{s}<2 / 3<\gamma_{-} \approx 1<\gamma_{+} \approx 4 / 3$ to show the transition of the universe from an early radiation dominated era to an intermediate dark matter dominated era (non-baryonic) to end in a dark energy dominated era at late times. Note that the integration constants $\mathcal{A}, \mathcal{B}$ and $\mathcal{C}$ can be esxpressed in terms of the density parameters $\Omega_{\mathrm{v} 0}=\rho_{\mathrm{v} 0} / 3 H_{0}^{2}, \Omega_{\mathrm{c} 0}=\rho_{\mathrm{c} 0} / 3 H_{0}^{2}, \Omega_{\mathrm{dr} 0}=\rho_{\mathrm{dr} 0} / 3 H_{0}^{2}$, and $\Omega_{\mathrm{r} 0}=\rho_{\mathrm{r} 0} / 3 H_{0}^{2}($ see $[9])$. The flatness condition reads $\Omega_{\phi 0}+\Omega_{\mathrm{c} 0}+\Omega_{\mathrm{r} 0}=1$ along with $\Omega_{\mathrm{v} 0}+\Omega_{\mathrm{dr} 0}=\Omega_{\phi 0}$.

We will now reconstruct the potential as a function of the scalar field $\phi$ by using that $V(a), \dot{\phi}^{2}(a)$ and $\rho_{t}(a)$ are explicit function of the scale factor through $V=\rho_{\mathrm{x}}$, $\dot{\phi}^{2} / 2=\rho_{\mathrm{dr}}$ and $\rho_{\mathrm{t}}=\dot{\phi}^{2} / 2+V+\rho_{\mathrm{m}}+\rho_{r 0} / a^{3 \gamma_{\mathrm{r}}}$. Rewritten $\dot{\phi}^{2} / 2$ as $\dot{\phi}^{2}=3 \rho_{t} \phi^{\prime 2}$, we easily obtain $\phi=\phi(a)$,

$$
\phi=\int \frac{\sqrt{6 \Omega_{\mathrm{sm}}}}{a} d a,
$$

where $\Omega_{\mathrm{sm}}=\dot{\phi}^{2} / 2 \rho_{\mathrm{t}}$. After integrate the Eq. (27), we find $\phi(a)$ and then follows $V(\phi)=\rho_{\mathrm{x}}(a(\phi))$. Also, the scalar field energy density $\rho_{\phi}=V+\dot{\phi}^{2} / 2=\rho_{\mathrm{x}}+\rho_{\mathrm{dr}}$ is easily calculated as a real function of the scale factor,

$$
\begin{gathered}
\rho_{\phi}=3 H_{0}^{2}\left[\left(\gamma_{s}-1\right)^{2} \mathcal{A} x^{3 \gamma_{s}}+\left(\gamma_{+}-1\right)^{2} \mathcal{B} x^{3 \gamma_{+}}\right. \\
\left.+\left(\gamma_{-}-1\right)^{2} \mathcal{C} x^{3 \gamma_{-}}\right] .
\end{gathered}
$$

We have changed the original degree of freedom $\phi$ by the scale factor $a$; such fact was essential to facilitate the reconstruction process and to examine the observational constraints through the Hubble expansion rate.

In the early radiation-dominated era, where $\gamma \simeq \gamma_{+} \approx$ $4 / 3$, and in the dark energy dominated era where the universe has an accelerated expansion implying $\gamma \simeq \gamma_{s}$, we obtain the approximate potential and scalar field,

$$
\begin{gathered}
V \simeq \frac{3 H_{0}^{2}}{2}\left(\gamma_{+}-2\right)\left(\gamma_{+}-1\right) \mathcal{B} a^{-3 \gamma_{+}} \\
\Delta \phi \simeq-\sqrt{3 \gamma_{+}\left(\gamma_{+}-1\right)} \ln a
\end{gathered}
$$

the former being negative definite and the latter becomes real, while $a \approx t^{2 / 3} \gamma_{+}$. Hence after having reconstructed the potential and the interaction term $Q_{\mathrm{m}}$, we obtain

$$
\begin{gathered}
V \simeq \frac{3 H_{0}^{2}}{2}\left(\gamma_{+}-2\right)\left(\gamma_{+}-1\right) \mathcal{B} e^{\sqrt{3 \gamma_{+} /\left(\gamma_{+}-1\right)} \Delta \phi}, \\
Q_{\mathrm{m}} \simeq-3 H_{0}^{2} \gamma_{+}\left(\gamma_{+}-2\right)\left(\gamma_{+}-1\right) \mathcal{B} e^{\sqrt{3 \gamma_{+} /\left(\gamma_{+}-1\right)} \Delta \phi},
\end{gathered}
$$

in the early epochs.

At late times, when the dark energy governs the dynamic of the universe, we find

$$
V \simeq \frac{3 H_{0}^{2}}{2}\left(\gamma_{s}-2\right)\left(\gamma_{s}-1\right) \mathcal{A} a^{-3 \gamma_{s}}
$$




$$
\Delta \phi \simeq \sqrt{3 \gamma_{s}\left(\gamma_{s}-1\right)} \ln a,
$$

hence, after having applied the reconstruction process, we find that the scalar field is driven by

$$
V \simeq \frac{3 H_{0}^{2}}{2}\left(\gamma_{s}-2\right)\left(\gamma_{s}-1\right) \mathcal{A} e^{-\sqrt{3 \gamma_{s} /\left(\gamma_{s}-1\right)} \Delta \phi},
$$

a positive potential because $\gamma \approx \gamma_{s}$. In this late regime $a \approx t^{2 / 3 \gamma_{s}}$ and the approximate $Q_{\mathrm{m}} \simeq-3 H_{0}^{2} \gamma_{s}\left(\gamma_{s}-\right.$ 2) $\left(\gamma_{s}-1\right) \mathcal{A} e^{\sqrt{3 \gamma_{s} /\left(\gamma_{s}-1\right)} \Delta \phi}$.

\section{OBSERVATIONAL CONSTRAINTS ON A TRANSVERSAL INTERACTING MODEL: SUMMARY AND DISCUSSION}

We will provide a qualitative estimation of the cosmological paramaters by constraining them with the Hubble data [17]- [18] and the stricts bounds for the behavior of dark energy at early times [11]-19]. In the former case, the statistical analysis is based on the $\chi^{2}$-function of the Hubble data [17], 18]. For model I, the Hubble expansion is given by

$$
H_{\mathrm{I}}(\theta \mid z)=H_{0}\left(\mathcal{A} x^{3 \gamma_{s}}+\mathcal{B} x^{4}+\mathcal{C} x^{3}\right)^{\frac{1}{2}} .
$$

Fron now on we will choose $\gamma_{+}=4 / 3$ and $\gamma_{-}=1$, so the parameters of the model are $\theta=\left\{H_{0}, \gamma_{s}, \Omega_{x 0}, \Omega_{m 0}\right\}$, and will use that the flatness condition implies $\Omega_{r 0}=$ $1-\Omega_{x 0}-\Omega_{m 0}$. We obtain that $\gamma_{s}$ varies from $10^{-4}$
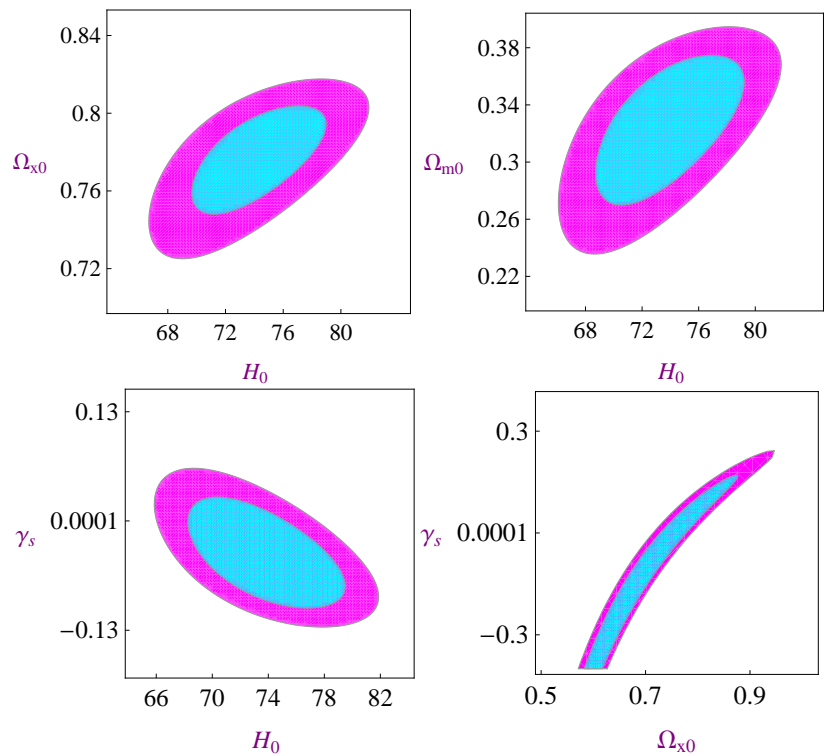

FIG. 2: Model I: two-dimensional C.L. associated with $1 \sigma, 2 \sigma$ for different $\theta$ planes.

to $10^{-3}$, so these values clearly fulfill the constraint $\gamma_{s}<2 / 3$ that assure the existence of accelerated phase of the universe at late times [see Fig. (2)]. We find the best-fit at $\left(H_{0}, \Omega_{x 0}\right)=\left(74.32 \mathrm{~km} \mathrm{~s}^{-1} \mathrm{Mpc}^{-1}, 0.77\right)$ with $\chi_{\text {d.o.f }}^{2}=0.783$ by using the priors $\Omega_{m 0}=0.2$ and $\gamma_{s}=10^{-3}$. The value of $\Omega_{x 0}$ is slightly greater than the standard one of 0.7 being such discrepancy only of $0.1 \%$. Moreover, we find that using the priors $\left(H_{0}, \gamma_{s}\right)=\left(74.20 \mathrm{~km} \mathrm{~s}^{-1} \mathrm{Mpc}^{-1}, 10^{-3}\right)$ the bestfit values for the present-day density parameters are $\left(\Omega_{x 0}, \Omega_{m 0}\right)=(0.74,0.23)$ along with a lower goodness condition $\left(\chi_{\text {d.o.f }}^{2}=0.779\right)$. In performing the statistical analysis, we find that $H_{0} \in[71.28,74.32] \mathrm{km} \mathrm{s}^{-1} \mathrm{Mpc}^{-1}$ so the estimated values are met within $1 \sigma$ C.L. reported by Riess et al, to wit, $H_{0}=(72.2 \pm 3.6) \mathrm{km} \mathrm{s}^{-1} \mathrm{Mpc}^{-1}$. We also find that the $z_{t}$ (transition redshift) is of the order unity varying over the interval $[0.63,0.75]$, such values are close to $z_{t}=0.69_{-0.13}^{+0.20}$ reported in [20]-[21].

In the case of model II, one finds that the Hubble expansion takes the following form:

$$
H_{\mathrm{II}}(\theta \mid z)=H_{0}\left(\mathcal{A} x^{3 \gamma_{s}}+\mathcal{B} x^{3 \gamma_{+}}+\mathcal{C} x^{3 \gamma_{-}}+\mathcal{D} x^{3 \gamma_{r}}\right)^{\frac{1}{2}} .
$$

Here, we consider $\theta=\left\{H_{0}, \gamma_{\mathrm{s}}, \gamma_{+}, \gamma_{-}, \gamma_{\mathrm{r}}, \Omega_{\mathrm{v} 0}, \Omega_{\mathrm{dr} 0}, \Omega_{\mathrm{c} 0}\right\}$ as the independent parameters to be constrained for the model encoded in the Hubble function (37). We start our statistical estimations by performing a global analysis on the eight parameters that characterize the model. In doing so, we find that $\chi^{2}(\theta)$ reaches a minimum at $\left\{H_{0}, \gamma_{\mathrm{s}}, \gamma_{+}, \gamma_{-}, \gamma_{\mathrm{r}}, \Omega_{\mathrm{v} 0}, \Omega_{\mathrm{dr} 0}, \Omega_{\mathrm{c} 0}\right\}=$ $(70.00,0.010,1.300,1.009,1.3400,0.749,0.00006,0.199)$ along with $\chi_{\text {d.o.f }}^{2}=20.172 /(29-8) \simeq 0.9605<1$. We see that $\gamma_{s}$ varies from $0.010_{-0.090-0.112}^{+0.007+0.055}$ to $0.010_{-0.189-0.303}^{+0.271+0.335}$ at $1 \sigma-2 \sigma$ confidence levels, so these values fulfill the constraint $\gamma_{s}<2 / 3$ at $95 \%$ C.L. that ensure the existence of accelerated phase of the universe at late times [ See Fig. (3)]. We find the best fit at $\left(H_{0}, \Omega_{\mathrm{v} 0}\right)=$ $\left(70.00_{-2.03-3.16}^{+4.99+6.07} \mathrm{~km} \mathrm{~s}^{-1} \mathrm{Mpc}^{-1}, 0.7299_{-0.0109-0.0238}^{+0.057+0.0658}\right)$

with $\chi_{\text {d.o.f }}^{2}=0.736$ by using the priors $\left(\Omega_{\mathrm{c} 0}, \Omega_{\mathrm{dr} 0}, \gamma_{s}, \gamma_{+}, \gamma_{-}, \gamma_{\mathrm{r}}\right)=(0.1999,6 \times$ $\left.10^{-5}, 0.010,1.3000,1.0099,1.3400\right)$. Moreover, we find that using the priors $\left(H_{0}, \Omega_{\mathrm{dr} 0}, \gamma_{s}, \gamma_{+}, \gamma_{-}, \gamma_{\mathrm{r}}\right)=\left(70.00 \mathrm{~km} \mathrm{~s}^{-1} \mathrm{Mpc}^{-1}, 6 \times\right.$ $\left.10^{-5}, 0.010,1.3000,1.0099,1.3400\right)$ the best-fit values for the present-day density parameters are considerably improved, namely, these turn give $\left(\Omega_{\mathrm{v} 0}, \Omega_{\mathrm{c} 0}\right)=\left(0.729_{-0.102-0.152}^{+0.167+0.218}, 0.239_{-0.281-0.364}^{+0.210+0.355}\right)$ along with the same goodness condition $\left(\chi_{\text {d.o.f }}^{2}=0.7368\right)$ Notice that $\Omega_{\mathrm{c} 0}$ varies from $0.239_{-0.019-0.042}^{+0.086+0.101}$ to $0.239_{-0.091-0.115}^{+0.215+0.267}$ at $68 \%, 95 \%$ C.L., whereas $H_{0} \in\left[70.00_{-2.04-3.05}^{+2.57+3.54}, 70.00_{-2.03-3.16}^{+4.99+6.07} \mathrm{~km} \mathrm{~s}^{-1} \mathrm{Mpc}^{-1}\right.$; thus, the latter values are consistent with the analysis of ACT and WMAP-7 data that gives $H_{0}=69.7 \pm 2.5 \mathrm{~km} \mathrm{~s}^{-1} \mathrm{Mpc}^{-1}$. We find that the transition redshift is $z_{\mathrm{t}}=0.604_{-0}^{+0.203+0.325}$ at $1 \sigma, 2 \sigma$ C.L., close to ones reported in [20], 21] or with the marginalized best-fit values $z_{\mathrm{t}}=0.623_{-0.052}^{+0.039}$ listed in [22], [23], 24].

The behavior of dark energy at early times can be considered as a new cosmological probe for testing dynamical dark energy models. The fraction of dark energy at recombination epoch should fulfill the bound 

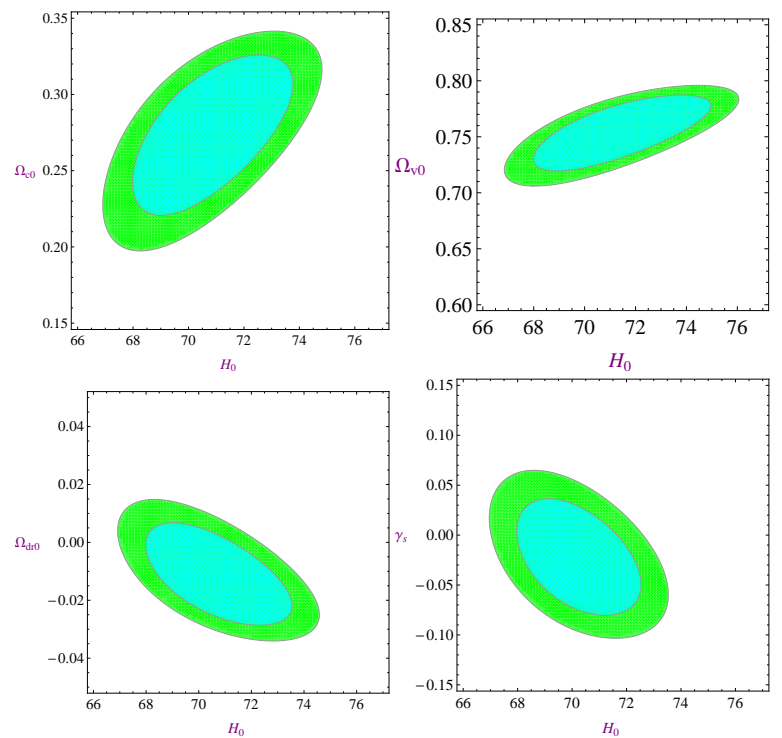

FIG. 3: Model II: two-dimensional C.L. associated with $1 \sigma, 2 \sigma$ for different $\theta$ planes.

$\Omega_{\text {ede }}(z \simeq 1100)<0.1$ in order to the dark energy model be consistent with the big-bang nucleosynthesis (BBN) data. Some signals could arise from the early dark energy (EDE) models uncovering the nature of DE as well as their properties to high redshift, giving an invaluable clue to the physics behind the recent speed up of the universe [11]. Then, it was examined the current and future data for constraining the amount of EDE, the cosmological data analyzed has led to an upper bound of $\Omega_{\text {ede }}(z \simeq 1100)<0.043$ with $95 \%$ confidence level (CL) in case of relativistic EDE while for a quintessence type of $\mathrm{EDE}$ has given $\Omega_{\mathrm{ede}}(z \simeq 1100)<0.024$ although the EDE component is not preferred, it is also not excluded from the current data [11]. Another forecast for the bounds of the EDE are obtained with the Planck and CMBPol experiments [19], thus assuming a $\Omega_{\text {ede }}\left(a \simeq 10^{-3}\right) \simeq 0.03$ for studying the stability of this value, it found that $1 \sigma$ error coming from Planck experiment is $\sigma_{\text {ede }}^{\text {Planck }} \simeq 0.004$ whereas the CMBPol improved this bound by a factor 4 [19].

For the model I, we find that at early times the dark energy changes rapidly with the redshift $z$ over the interval $\left[10^{3}, 10^{4}\right]$, indeed, around $z \simeq 1100$ we find that $2.4 \times$ $10^{-11} \leq \Omega_{\mathrm{x}} \leq 2.6 \times 10^{-10}$. However for the model II, we find that the dark energy around $z \simeq 1100, \Omega_{\phi} \simeq 0.014$. In comparison with the SPT constraints on the early dark energy density over WMAP7 alone it was found that the $95 \%$ upper limit on $\Omega_{\text {ede }}$ is reduced from 0.052 for WMAP7-only to 0.013 for WMAP7+SPT. This is a $38 \%$ improvement on the upper limit of $\Omega_{\text {ede }}<0.018$ reported for WMAP7+K11 25]. The upper limit is essentially unchanged at $\Omega_{\text {ede }}<0.014$ for $\mathrm{WMAP} 7+\mathrm{SPT}+\mathrm{BAO}+\mathrm{SNe}$. The $\Omega_{\text {ede }}<0.013$ bound from WMAP + SPT is the best published constraint from the CMB (see [26] and reference therein). Our findings point out that the model constructed here not only fulfill the severe bound of $\Omega_{\phi}(z \simeq 1100)<0.018$ obtained from the measurements of CMB anisotropy from ACT and SPT [25], [12], 26] but also is consistent with the future constraints achievable by Planck and CMBPol experiments [19] as well, corroborating that the value of the cosmological parameters selected before, through the statistical analysis made with Hubble data, are consistent with BBN constraints. Besides, regarding the values reached by dark energy around $z=10^{10}(\mathrm{BBN})$, we find that $\Omega_{\phi}=0.0025$ at $1 \sigma$ level indicating the conventional BBN processes that occurred at temperature of $1 \mathrm{Mev}$ is not spoiled because the severe bound reported for early dark energy $\Omega_{\phi}\left(z \simeq 10^{10}\right)<0.21[13]$ or a strong upper limit $\Omega_{\phi}\left(z \simeq 10^{10}\right)<0.04[14]$ are fulfilled at BBN.

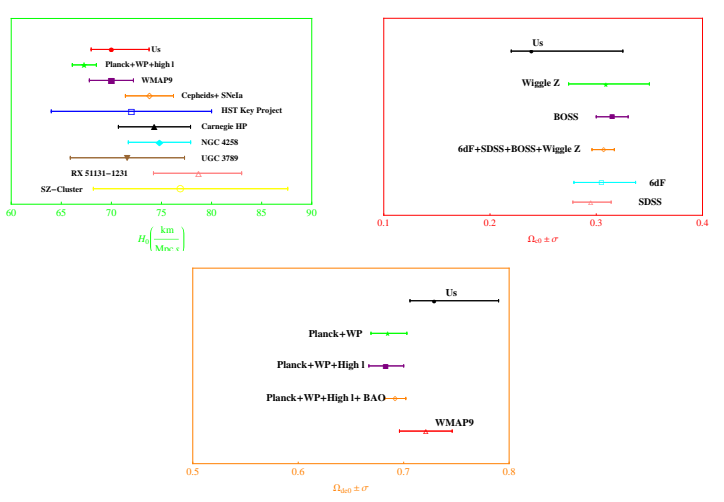

FIG. 4: Comparison of $H_{0}, \Omega_{\mathrm{c} 0}$, and $\Omega_{\mathrm{v} 0}$ measurements, with estimates of $\pm 1 \sigma$ errors, from a number of different methods. These are compared with the data provided by Planck and WMAP9.

We will perform a further comparative analysis by taking into account several data sets along with theirs constraints on the most relavant parameters [15], [16], namely, the values or bounds reported for $H_{0}, \Omega_{\mathrm{v} 0}$, $\Omega_{\mathrm{c} 0}$, and $\Omega_{\mathrm{ede}}$. The Planck power spectra leads to a low value of the Hubble constant, which is tightly constrained by CMB data alone within the $\Lambda$ CDM model. From the Planck $+\mathrm{WP}+$ highL analysis, it found that $H_{0}=(67.3 \pm 1.2) \mathrm{kms}^{-1} \mathrm{Mpc}^{-1}$ at $68 \%$ [15]. A low value of $H_{0}$ has been found in other CMB experiments, most notably from the recent WMAP-9 analysis. Fitting the base $\Lambda$ CDM model for the WMAP-9 data, it is found $H_{0}=(70.0 \pm 2.2) \mathrm{kms}^{-1} \mathrm{Mpc}^{-1}$ at $68 \%$ C.L. [16]. Then, our best estimation $H_{0}=70.00_{-2.02}^{+3.76} \mathrm{kms}^{-1} \mathrm{Mpc}^{-1}$ at $68 \%$ C.L is perfectly in agreement with the value reported by WMAP-9 project but shows a slightly difference with the Planck+WP+highL data, which is less than $0.04 \%$. In Fig. (4), we show other observational estimations of the Hubble constant that includes the megamaser-based distance to NGC4258, Cepheid observations, a revised distance to the Large Magellanic Cloud, and so on (see [15]). For dark matter, Wiggle-Z data give $\Omega_{\mathrm{c} 0}=0.309_{-0.035}^{+0.041}$, while Boss experiment increases dark matter amount in $0.019 \%$; the joint statistical analysis with $6 \mathrm{dF}+\mathrm{SDSS}+\mathrm{BOSS}+$ Wiggle-Z data lead to $\Omega_{\mathrm{c} 0}=0.307_{-0.011}^{+0.010}$ at $1 \sigma$ level [15], showing a discrepancy 
with our estimation of dark matter, $\Omega_{\mathrm{c} 0}=0.239_{-0.019}^{+0.086}$, no bigger than $0.24 \%$ [see Fig. (4)]. For the fraction of dark energy, Planck $+\mathrm{WP}$ data give $0.685_{-0.016}^{+0.018}$ at $68 \%$ C.L, whereas the joint statistical analysis on Planck $+\mathrm{WP}+$ highL $+\mathrm{BAO}$ gives $0.692 \pm 0.010$ at $1 \sigma$ level [15]. In our case, we find that $\Omega_{\mathrm{v} 0}=0.7299_{-0.0109}^{+0.057}$, then the relative difference between Hubble data and Planck+WP+highL is $0.068 \%$ [see Fig. (4)]. Besides, the $\mathrm{CMB}$ anisotropies measurements put further constraints on the behavior of dynamical dark energy in the recombination epoch; the latest constraints on early dark energy come from Planck $+\mathrm{WP}+$ highL data and leads to $\Omega_{\text {ede }}<0.009$ at $95 \%$ C.L [15]. We have found that $\Omega_{\phi}\left(z \simeq 10^{3}\right) \simeq 0.014$, so the relative error between both estimations is $\left|\Omega_{\phi}^{\mathrm{Us}}-\Omega_{\text {ede }}^{\text {Planck }}\right| /\left|\Omega_{\text {ede }}^{\text {Planck }}\right| \simeq 0.5 \%$. The latter disagreement can be reduced in a future by including additional measurements of Hubble data points or other data sets [27], allowing to improve the statistical analysis performed here.

\section{Acknowledgments}

L.P.C thanks UBA under Project No. 20020100100147 and CONICET under Project PIP 114-200801-00328 for their partial support. M.G.R is supported by Postdoctoral Fellowship program of CONICET.
[1] L.P.Chimento, Phys.Rev.D81 043525 (2010).

[2] M. Jamil, E. N. Saridakis, and M. R. Setare, Phys. Rev. D 81023007 (2010); M. Jamil, D. Momeni, M.A. Rashid, [arXiv: 1107.1558v2];

[3] Luis P. Chimento, Martín G. Richarte, Iván E. S. García, Phys. Rev. D 88087301 (2013).

[4] L. P. Chimento, A. S. Jakubi, D. Pavón, W. Zimdahl, Phys.Rev. D 67 (2003) 083513; W. Zimdahl, D. Pavón, L.P. Chimento, Phys.Lett. B 521 (2001) 133-138; F.E.M. Costa, E.M. Barboza, Jr., J.S. Alcaniz, Phys.Rev. D 79 (2009) 127302; P.C. Ferreira, J.C. Carvalho, J.S. Alcaniz, Phys.Rev. D 87 (2013) 8, 087301; Timothy Clemson, Kazuya Koyama, Gong-Bo Zhao, Roy Maartens, Jussi Valiviita, Phys.Rev. D85 (2012) 043007; Luca Amendola, Phys.Rev. D 69 (2004) 103524; S. del Campo, R. Herrera, G. Olivares, D. Pavón, Phys.Rev. D74 (2006) 023501; John D. Barrow, T. Clifton, Phys.Rev. D 73 (2006) 103520; Jussi Valiviita, Elisabetta Majerotto, Roy Maartens, JCAP 0807 (2008) 020; S. del Campo, R. Herrera, D. Pavón, JCAP 0901 (2009) 020; Hao Wei, Phys.Lett. B 691 (2010) 173-182;Julio C. Fabris, Bernardo Fraga, Nelson Pinto-Neto, Winfried Zimdahl, JCAP 1004 (2010) 008; M. Forte, [e-Print: arXiv:1311.3921.

[5] L. P. Chimento, M. Forte, R. Lazkoz and M. G. Richarte, Phys.Rev.D 79043502 (2009).

[6] L. P. Chimento, M. G. Richarte, Phys.Rev. D 84123507 (2011).

[7] L. P. Chimento, M. G. Richarte, Phys.Rev. D 85127301 (2012).

[8] Luis P. Chimento and Martín G. Richarte, Phys. Rev. D 86103501 (2012).

[9] L. P. Chimento, M. G. Richarte, Eur. Phys. J. C 73 (2013) 2497.

[10] Kai Liao, Zhengxiang Li, Jing Ming, Zong-Hong Zhu, Phys. Lett. B 718 (2013) 1166-1170; O. farooq and B. Ratra, arXiv:1301.5243; M. Moresco, L. Verde, L. Pozzetti, R. Jimenez and A. Cimatti, JCAP 1207, 053 (2012).

[11] E. Calabrese, D. Huterer, E. V. Linder, A. Melchiorri and L.Pagano, Phys.Rev.D 83123504 (2011).

[12] V. Pettorino, L. Amendola, C. Wetterich, arXiv:1301.5279
[13] R.H. Cyburt, B.D. Fields, K. A. Olive and E. Skillman, Astropart. Phys. 23, 313 (2005).

[14] E.L. Wright, The Astrophysical Journal, 664 633-639, 2007.

[15] P. A. R. Ade et al, arXiv:1303.5076v1].

[16] G. Hinshaw et al., arXiv:1212.5226v3].

[17] J. Simon, L. Verde and R. Jimenez, Phys. Rev. D 71 123001 (2005) astro-ph/0412269; L. Samushia and B. Ratra, Astrophys. J. 650, L5 (2006).

[18] D. Stern et al., arXiv:0907.3149. E. Calabrese, M. Archidiacono, A. Melchiorri, B. Ratra, arXiv:1205.6753.

[19] E. Calabrese, R. de Putter, D. Huterer, E. V. Linder, A. Melchiorri, Phys.Rev.D 83023011 (2011).

[20] J. Lu, L. Xu and M. Liu, Physics Letters B 699, 246 (2011).

[21] Mónica I. Forte, Martín G. Richarte, arXiv:1206.1073; Luis P. Chimento, Mónica I. Forte, Martín G. Richarte, arXiv:1206.0179; Luis P. Chimento, Mónica Forte, Martín G. Richarte, arXiv:1106.0781 ]; Luis P. Chimento, Martín G. Richarte, arXiv:1207.1121.

[22] L. P. Chimento, M. G. Richarte, [arXiv:1303.3356].

[23] L. P. Chimento, M.I. Forte, M. G. Richarte, arXiv:1301.2737]; L. P. Chimento, M.I. Forte, M. G. Richarte, arXiv:1106.0781.

[24] J. A. S. Lima, J. F. Jesus, R. C. Santos, M. S. S. Gill, arXiv:1205.4688]

[25] Christian L. Reichardt, Roland de Putter, Oliver Zahn, Zhen Hou, arXiv:1110.5328.

[26] Z.Hou et al, [arXiv: 1212.6267]

[27] Lixin Xu, Yuting Wang, Hyerim Noh, Eur. Phys. J. C (2012) 72, 1931;Lixin Xu, Physical Review D 85, 123505 (2012); Lixin Xu, Phys. Rev. D. 87, 043503(2013); Yuting Wang, David Wands, Lixin Xu, Josue De-Santiago, Alireza Hojjati, Phys. Rev. D 87, 083503 (2013); Lixin Xu, Phys. Rev. D. 87, 043525(2013); Lixin Xu, Phys. Rev. D 88, 084032 (2013); Lixin Xu, Yadong Chang, Phys. Rev. D 88, 127301 (2013);Weiqiang Yang, Lixin $\mathrm{Xu}$, [arXiv:1311.3419]; Weiqiang Yang, Lixin Xu, Phys. Rev. D 88, 023505 (2013); Weiqiang Yang, Lixin Xu, Yuting Wang, Yabo Wu, [ arXiv:1312.2769]; Weiqiang Yang, Lixin Xu, [arXiv:1401.1286], [arXiv:1401.5177]. 